\title{
Communication \\ Two-Phonon Blockade in Quadratically Coupled Optomechanical Systems
}

\author{
Zi-Yuan Li, Guang-Ri Jin, Tai-Shuang Yin and Aixi Chen *
}

Department of Physics, Zhejiang Sci-Tech University, Hangzhou 310018, China; 201920109009@mails.zstu.edu.cn (Z.-Y.L.); grjin@zstu.edu.cn (G.-R.J.); taishuangyin@zstu.edu.cn (T.-S.Y.)

* Correspondence: aixichen@zstu.edu.cn

Citation: Li, Z.-Y.; Jin, G.-R.; Yin,

T.-S.; Chen, A. Two-Phonon Blockade

in Quadratically Coupled

Optomechanical Systems. Photonics

2022, 9, 70. https://doi.org/

$10.3390 /$ photonics 9020070

Received: 3 January 2022

Accepted: 24 January 2022

Published: 27 January 2022

Publisher's Note: MDPI stays neutral with regard to jurisdictional claims in published maps and institutional affiliations.

Copyright: (C) 2022 by the authors. Licensee MDPI, Basel, Switzerland. This article is an open access article distributed under the terms and conditions of the Creative Commons Attribution (CC BY) license (https:// creativecommons.org/licenses/by/ $4.0 /)$.

\begin{abstract}
We propose a scheme to realize the two-phonon blockade effect in a quadratically coupled optomechanical system. We consider the case that the optical cavity is simultaneously driven by a strong pumping field and a weak driving field. By strongly driving the optical cavity, the nonlinear interaction between the optical mode and the mechanical resonator can be significantly enhanced and an effective second-order nonlinearity between photons and phonons is induced. Based on this effectively strong nonlinearity, the two-phonon blockade effect can be achieved when a weak driving field is applied into the optical cavity. By contrast, we study the case of weakly driving the mechanical resonator. In this case, the single-phonon blockade is generated, while the two-phonon blockade cannot be observed. By numerically calculating the second-order and third-order correlation function, we investigate the statistical characteristics of phonons. In addition, we also study the influence of the thermal noise on the achieved two-phonon blockade effect. Our work provides an alternative approach for implementing multiphonon blockade and has potential applications in quantum information processing.
\end{abstract}

Keywords: quadratic optomechanical coupling; strong nonlinearity; two-phonon blockade

\section{Introduction}

In recent years, cavity optomechanics, exploring the interaction between optical and mechanical degrees of freedom [1-5], has received extensive attention and made spectacular achievements. For example, the mechanical resonators with a wide range of frequencies could be experimentally cooled to their quantum ground state [6-10]. This achievement offers the possibility of manipulating and controlling the mechanical resonators at the singlephonon level. In particular, many theoretical and experimental efforts have been devoted to enhancing the intrinsically weak optomechanical nonlinearity [11-17] so that the singlephoton quantum regime can be realized. This quantum regime allows for many interesting quantum effects, including macroscopic nonclassical states [18-22], the photon blockade phenomenon [23-27] and the multiphonon sideband effects [28]. Most of these works focus on the linear optomechanical coupling, i.e., an optical mode is parametrically coupled to the position of a mechanical oscillator. Note that many recent works study a quadratically coupled optomechanical system [29-32], where an optical mode is parametrically coupled to the square of the position of a mechanical oscillator. The quadratic optomechanical coupling has been utilized to realize the quantum nondemolition measurements of the mechanical oscillator's energy eigenstates [29], to achieve the cooling and squeezing of the mechanical oscillator [33,34], to generate the photon blockade $[27,35]$, the phonon antibunching and phonon blockade [36-39].

Phonon blockade, as a typical quantum effect, has attracted a lot of attention and has been extensively studied [36-46]. In analogy to photon blockade, phonon blockade presents a quantum phenomenon in which the appearance of one phonon prevents the excitation of the second phonon in a nonlinear mechanical oscillator. The generation of a 
phonon blockade generally requires a strong mechanical nonlinearity, which could induce the energy-level anharmonicity of a system. This required mechanical nonlinearity can be realized in quadratically coupled optomechanical systems [36-39] or in systems with the nanomechanical resonator coupled to a qubit [40-42] or an intrinsic two-level system defect [45]. The quantum effect of phonon blockade plays a crucial role not only in revealing the quantum behaviors of a mechanical system, but also in promoting the development of single-phonon quantum devices as well as their applications in quantum information processing [21].

Here we propose a scheme to realize the two-phonon blockade effect in a quadratically coupled optomechanical system. To clearly understand the two-phonon blockade, one can call the typical phonon blockade that the majority of works aim at as the single-phonon blockade. Similar to the single-phonon blockade, the two-phonon blockade means that the resonant absorption of two phonons will impede the transmission of the subsequent phonons. To achieve the two-phonon blockade, we consider that the optical cavity is both driven by a strong pumping field and a weak driving field. By strongly driving the optical cavity, the nonlinear interaction between the optical mode and the mechanical mode can be significantly enhanced and can be tuned by adjusting the amplitude of the driving field. This strong nonlinear interaction leads to the energy-level anharmonicity of the system. In addition, an effective second-order nonlinearity between photons and phonons is induced. This effectively strong nonlinearity ultimately allows for the generation of the two-phonon blockade effect when a weak driving field is applied into the optical cavity. We investigate the statistical characteristics of phonons by numerically calculating the second-order and third-order correlation function. As a comparison, we also study the case of weakly driving the mechanical resonator. In this case, the results show that the single-phonon blockade occurs, while the two-phonon blockade can not be obtained. Furthermore, we also study the influence of the thermal noise on the achieved two-phonon blockade effect. The twophonon blockade effect can be exploited to achieve two-phonon sources and may inspire the implementation of multiphonon quantum devices. Currently, few works study this quantum effect. Therefore, our work provides an alternative approach for implementing miltiphonon blockade and has potential applications in quantum information processing.

Note that some recent works have proposed to investigate the phonon antibunching and phonon blockade in quadratically coupled optomechanical systems. Our work is different from these works in several respects. First, we employ quadratic optomechanical coupling to achieve the two-phonon blockade effect instead of the single-phonon blockade [36-39] or photon blockade and photon-induced tunneling [27]. Second, in our proposal, the two-phonon blockade effect is based on the effectively enhanced second-order nonlinearity between photons and phonons. In contrast, the work in Ref. [36] exploits an effective cubic nonlinearity for the mechanics by adiabatic elimination of the cavity field, while the work in Ref. [37] utilizes the Kerr-type nonlinear interaction between phonons to analyze the single-phonon blockade. In addition, by applying two driving fields into the optical cavity, we illustrate the quantum effects of phonons, while Ref. [27] explores single-photon nonlinearities in a strongly driven optomechanical system.

\section{Model and Hamiltonian}

We study a quadratically coupled optomechanical system with a "membrane-in-themiddle" configuration, as schematically shown in Figure 1a. By placing the membrane in the node (or antinode) of the standing wave in the cavity [29,30], the cavity field will quadratically couple to the displacement of the mechanical motion. In addition, the optical cavity is driven by a strong pumping field with frequency $\omega_{L}$ and amplitude $\Omega$. The system Hamiltonian reads $(\hbar=1)$

$$
H=\omega_{c} a^{\dagger} a+\omega_{m} b^{\dagger} b+g_{0} a^{\dagger} a\left(b+b^{\dagger}\right)^{2}+H_{d},
$$


where $a\left(a^{\dagger}\right)$ and $b\left(b^{\dagger}\right)$ are the annihilation (creation) operators of the cavity mode and mechanical mode, respectively. The first term in Equation (1) represents is the Hamiltonian of the cavity field with frequency $\omega_{c}$. The second term is the free energy of the mechanical oscillator with frequency $\omega_{m}$. The third term describes the quadratic optomechanical coupling with the interaction strength $g_{0}$. The last term $H_{d}$ denotes the coupling between the cavity and the strong pumping field with $H_{d}=\Omega\left(a^{\dagger} e^{-i \omega_{L} t}+a e^{i \omega_{L} t}\right)$.

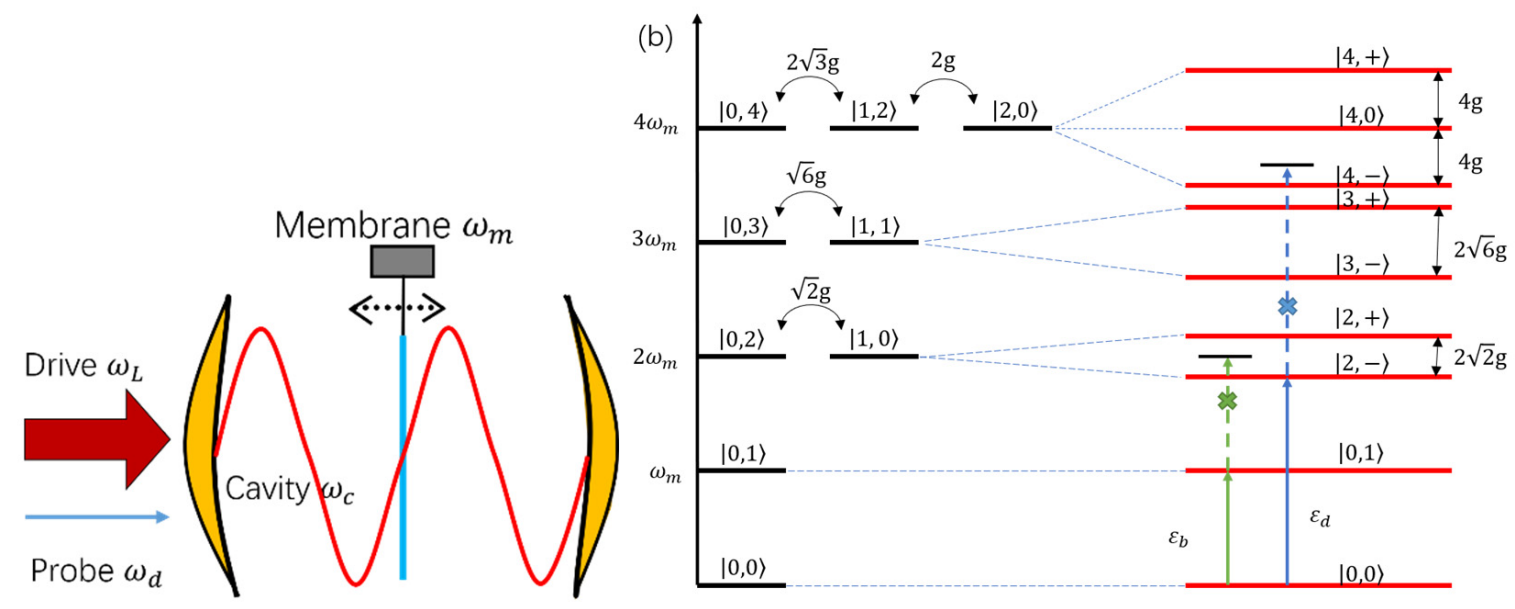

Figure 1. (a) Schematic illustration of an optomechanical system consisting of an optical cavity mode (with frequency $\omega_{c}$ ) quadratically coupled to the mechanical mode (with frequency $\omega_{m}$ ) with the "membrane-in-the-middle" configuration. In addition, the optical cavity is driven by a strong pumping field with frequency $\omega_{L}$ and a weak driving field with frequency $\omega_{d}$. (b) Energy-level diagram of the quadratically coupled optomechanical system. Here the bare states are labelled by $|n, m\rangle$, where $|n\rangle$ and $|m\rangle$ denote the eigenstates of the photon number and the phonon number, respectively. The green arrow describes the process of the single-phonon blockade when the mechanical resonator is weakly driven with the driving strength $\varepsilon_{b}$. The blue arrow describes the process of the two-phonon blockade when the optical cavity is weakly driven with the driving strength $\varepsilon_{d}$.

In a frame rotating with the driving frequency $\omega_{L}$, the system Hamiltonian can be written as

$$
H_{1}=\Delta_{c} a^{\dagger} a+\omega_{m} b^{\dagger} b+g_{0} a^{\dagger} a\left(b+b^{\dagger}\right)^{2}+\Omega\left(a^{\dagger}+a\right),
$$

where $\Delta_{c}=\omega_{c}-\omega_{L}$ is the frequency detuning between the cavity mode and the driving field.

For a sufficiently strong driving field, one can decompose the field operators $a$ and $b$ as the sum of their average amplitudes and small quantum fluctuations, i.e., $a \rightarrow \alpha+a$, $b \rightarrow \beta+b$. By solving the Heisenberg-Langevin equations, the steady-state classical amplitudes $\alpha$ and $\beta$ can be obtained as $\alpha=\Omega /\left(i \kappa / 2-\Delta_{c}\right)$ and $\beta=0$, where $\kappa$ is the cavity dissipation rate. The amplitude $\alpha$ is real for a proper choice of the phase of $\Omega$. Following the standard linearization procedure, the Hamiltonian in Equation (2) becomes $H_{2}=\Delta_{c} a^{\dagger} a+$ $\omega_{m} b^{\dagger} b+g\left(a^{\dagger}+a\right)\left(b+b^{\dagger}\right)^{2}$, where $g=g_{0} \alpha$ is the effective coupling strength which can be enhanced and tuned by controlling the amplitude of the driving field. Note that here we have neglected the term $g_{0} a^{\dagger} a\left(b+b^{\dagger}\right)^{2}$ in the Hamiltonian $H_{2}$ due to the small influence in the case of $|\alpha| \gg 1$. We consider that the external driving field satisfies the red-detuned two-phonon resonant condition, i.e., $\Delta_{c}=2 \omega_{m}$. Then, under the condition of $\omega_{m} \gg g$, the terms that oscillate with high frequencies $\pm \Delta_{c}, \pm\left(\Delta_{c}+\omega_{m}\right)$ can be safely neglected under the rotating-wave approximation (RWA). As a result, the system Hamiltonian can be approximately written as

$$
H^{\prime}=\Delta_{c} a^{\dagger} a+\omega_{m} b^{\dagger} b+g\left(a^{\dagger} b^{2}+a b^{\dagger 2}\right) .
$$


Here we obtain an effective second-order nonlinearity between photons and phonons with the significantly enhanced coupling strength $g$, which can be much larger that the optical dissipation $\kappa$. On the one hand, this nonlinear interaction will lead to a process that the annihilation (creation) of a photon is accompanied by the creation (annihilation) of two phonons. On the other hand, in the strong coupling regime $(g \gg \kappa)$, one can obtain the energy-level anharmonicity of the system, as shown in Figure 1b. The preferential basis is given by states of the form $|n, m\rangle$, where $n$ and $m$ denote the number of quanta in the cavity mode and mechanical mode, respectively. In the presence of the strong nonlinear interaction, the ground state of the coupled system is $|0,0\rangle$ and the first excited state is $|0,1\rangle$. However, the second excited state is the superposition state of $|0,2\rangle$ and $|1,0\rangle$ with the energy splitting $2 \sqrt{2} g$. The third excited state is the superposition state of $|0,3\rangle$ and $|1,1\rangle$ with the energy splitting $2 \sqrt{6} g$. In the fourth excitation manifold, the eigenstate is the superposition state of $|0,4\rangle,|1,2\rangle$ and $|2,0\rangle$. In the following text, we will explain the quantum phenomenon of the two-phonon blockade effect from the perspective of the energy level diagram.

Including the dissipation caused by the system-bath coupling, the dissipative dynamics of the system is described by the master equation

$$
\dot{\rho}=-i\left[H_{\mathrm{eff}}, \rho\right]+\kappa \mathcal{D}[a] \rho+\gamma n_{\mathrm{th}} \mathcal{D}[b] \rho+\gamma\left(n_{\mathrm{th}}+1\right) \mathcal{D}\left[b^{\dagger}\right] \rho,
$$

where $\mathcal{D}[o] \rho=o \rho o^{\dagger}-\frac{1}{2}\left(o^{\dagger} o \rho+\rho o^{\dagger} o\right)$ is the standard Lindblad superoperator, $\gamma$ is the decay rate of the mechanical oscillator, and $n_{\text {th }}$ is the thermal phonon occupation number with $n_{\mathrm{th}}=\left[\exp \left(\hbar \omega_{m} / k_{B} T\right)-1\right]^{-1}$, where $T$ is the temperature of the thermal reservoir.

To exhibit the quantum behavior of the phonons, we investigate the statistical properties of the phonons, which can be characterized by the steady-state second-order correlation function

$$
g^{(2)}(\tau)=\operatorname{Lim}_{t \rightarrow \infty} \frac{\left\langle b^{\dagger}(t) b^{\dagger}(t+\tau) b(t+\tau) b(t)\right\rangle}{\left\langle b^{\dagger}(t) b(t) b^{\dagger}(t+\tau) b(t+\tau)\right\rangle},
$$

and the steady-state third-order correlation function

$$
g^{(3)}\left(\tau_{1}, \tau_{2}\right)=\operatorname{Lim}_{t \rightarrow \infty} \frac{\left\langle b^{\dagger}(t) b^{\dagger}\left(t+\tau_{1}\right) b^{\dagger}\left(t+\tau_{2}\right) b\left(t+\tau_{2}\right) b\left(t+\tau_{1}\right) b(t)\right\rangle}{\left\langle b^{\dagger}(t) b(t)\right\rangle^{3}} .
$$

We assume the case of zero-delay between phonons, i.e., $\tau=\tau_{1}=\tau_{2}=0$. Then the equal-time second-order and third-order correlation functions in the steady state can be expressed as

$$
g^{(n)}(0)=\operatorname{Lim}_{t \rightarrow \infty} \frac{\left\langle b^{\dagger n} b^{n}\right\rangle(t)}{\left\langle b^{\dagger} b\right\rangle^{n}(t)},
$$

for $n=2,3$. Typically, $g^{(2)}(0)<1$ characterizes the antibunching effect of phonons with subPoisson statistics of phonons, and $g^{(2)}(0) \rightarrow 0$ reveals the generation of the single-phonon blockade effect. The two-phonon blockade effect occurs when $g^{(2)}(0)>1$ and $g^{(3)}(0)<1$.

\section{Two-Phonon Blockade via Weakly Driving the Optical Cavity}

To investigate the phonon statistics, a weak driving field needs to be applied into the system. Note that when the mechanical resonator is weakly driven, the single-photon blockade effect and photon-induced tunneling can be achieved, which has been shown in the work [27]. In order to study whether the two-phonon blockade can be generated in this case and compare with the case of weakly driving the optical cavity in the following text, here we first consider the case of weakly driving the mechanical mode with the Hamiltonian $H_{p}=\varepsilon_{b}\left(b^{\dagger} e^{-i \omega_{p} t}+b e^{i \omega_{p} t}\right)$. In a frame rotating with frequency $\omega_{p}$ for the mechanical mode and $2 \omega_{p}$ for the cavity mode, the effective Hamiltonian can be written as 
$H_{\text {eff }}=\Delta a^{\dagger} a+\Delta_{m} b^{\dagger} b+g\left(a^{\dagger} b^{2}+a b^{\dagger 2}\right)+\varepsilon_{b}\left(b^{\dagger}+b\right)$, where the detunings are $\Delta=\Delta_{c}-2 \omega_{p}$ and $\Delta_{m}=\omega_{m}-\omega_{p}$.

In Figure $2 a, b$, we calculate the steady-state equal-time second-order correlation function $g^{(2)}(0)$ and third-order correlation function $g^{(3)}(0)$ versus the detuning $\Delta_{m} / \kappa$ and the coupling strength $g / \kappa$ by numerically solving the master equation in Equation (4) with the effective Hamiltonian $H_{\text {eff }}$. It is shown that in the case of single-phonon resonant driving, i.e., $\Delta_{m}=0$, one can obtain the single-phonon blockade effect with $g^{(2)}(0) \ll 1$ and $g^{(3)}(0) \ll 1$. This phenomenon can be illustrated by the energy-level diagram in Figure $1 \mathrm{~b}$. If the driving laser is on resonance with the $|0,0\rangle \rightarrow|0,1\rangle$ transition, i.e., $\Delta_{m}=0$, the same $|0,1\rangle \rightarrow|2, \pm\rangle$ transition is detuned and will be suppressed for $g \gg \kappa, \gamma$. This could induce the generation of the single-phonon blockade effect, as indicated by the green arrow in Figure 1b. However, in the case of two-phonon resonant driving, i.e., $\Delta_{m}= \pm \frac{\sqrt{2}}{2} g$, the second-order correlation function satisfies $g^{(2)}(0) \gg 1$, as shown by the white dashed lines in Figure $2 a$, while $g^{(3)}(0) \gg 1$ at this detuning as seen from Figure $2 b$, which indicates the phonon-induced tunneling. In order to clearly present these results, in Figure $3 a$, the second-order correlation function $g^{(2)}(0)$ and third-order correlation function $g^{(3)}(0)$ are plotted as a function of the detuning $\Delta_{m} / g$. It is obviously seen that when the detuning is $\Delta_{m}=0$, the single-phonon blockade effect occurs with $g^{(2)}(0) \rightarrow 0$ and $g^{(3)}(0) \rightarrow 0$. When the detuning is $\Delta_{m}= \pm \frac{\sqrt{2}}{2} g, g^{(2)}(0) \gg 1$ and $g^{(3)}(0) \gg 1$, which is consistent with the results in Figure 2a,b. In addition, in Figure $3 \mathrm{~b}$ we plot $g^{(2)}(0)$ and $g^{(3)}(0)$ versus the driving strength $\varepsilon_{b} / \kappa$. One can find that when the driving strength $\varepsilon_{b} / \kappa$. varies in a wide range, e.g., from 0 to 2 , the values of $g^{(2)}(0)$ and $g^{(3)}(0)$ is always bigger than 1 . Therefore, these results suggest that one cannot achieve the two-phonon blockade effect when a weak driving field is applied into the mechanical resonator.
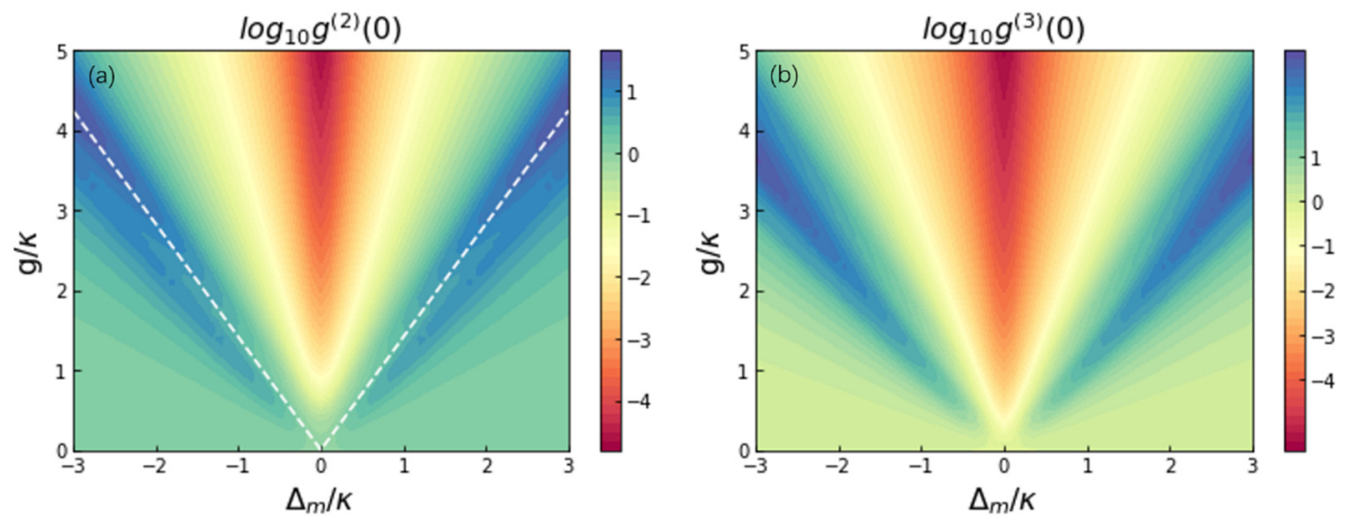

Figure 2. Steady-state equal-time (a) second-order correlation function $g^{(2)}(0)$ and (b) third-order correlation function $g^{(3)}(0)$ versus the detuning $\Delta_{m} / \kappa$. and the coupling strength $g / \kappa$ when the mechanical resonator is weakly driven. The system parameters we take are $\gamma=0.01 \kappa, \varepsilon_{b}=0.15 \kappa$ and $n_{\mathrm{th}}=0$. The white dashed curve in (a) corresponds to the case of two-phonon resonant driving, i.e., $\Delta_{m}= \pm \frac{\sqrt{2}}{2} g$.

To observe the two-phonon blockade effect, we consider a weak driving field (with frequency $\omega_{d}$ and strength $\varepsilon_{d}$ ) applied into the cavity with the Hamiltonian $H_{d c}=$ $\varepsilon_{d}\left(a^{\dagger} e^{-i \omega_{d} t}+a e^{i \omega_{d} t}\right)$. In the frame rotating with frequency $\omega_{L}$, the Hamiltonian of the driving field becomes $H_{d}^{\prime}=\varepsilon_{d}\left(a^{\dagger} e^{-i \delta t}+a e^{i \delta t}\right)$ with $\delta=\omega_{d}-\omega_{L}$. In a frame rotating with frequency $\delta$ for the cavity mode and $\delta / 2$ for the mechanical mode, the effective Hamiltonian can be written as

$$
H_{\mathrm{eff}}=\Delta^{\prime} a^{\dagger} a+\Delta_{m}^{\prime} b^{\dagger} b+g\left(a^{\dagger} b^{2}+a b^{\dagger 2}\right)+\varepsilon_{d}\left(a^{\dagger}+a\right),
$$

where the detunings are $\Delta^{\prime}=\Delta_{c}-\delta=\omega_{c}-\omega_{d}$ and $\Delta_{m}^{\prime}=\omega_{m}-\omega_{d} / 2$. In the following text, we calculate the steady-state equal-time second-order correlation function $g^{(2)}(0)$ 
and third-order correlation function $g^{(3)}(0)$ by numerically solving the master equation in Equation (4) but with the effective Hamiltonian $H_{\text {eff }}$.
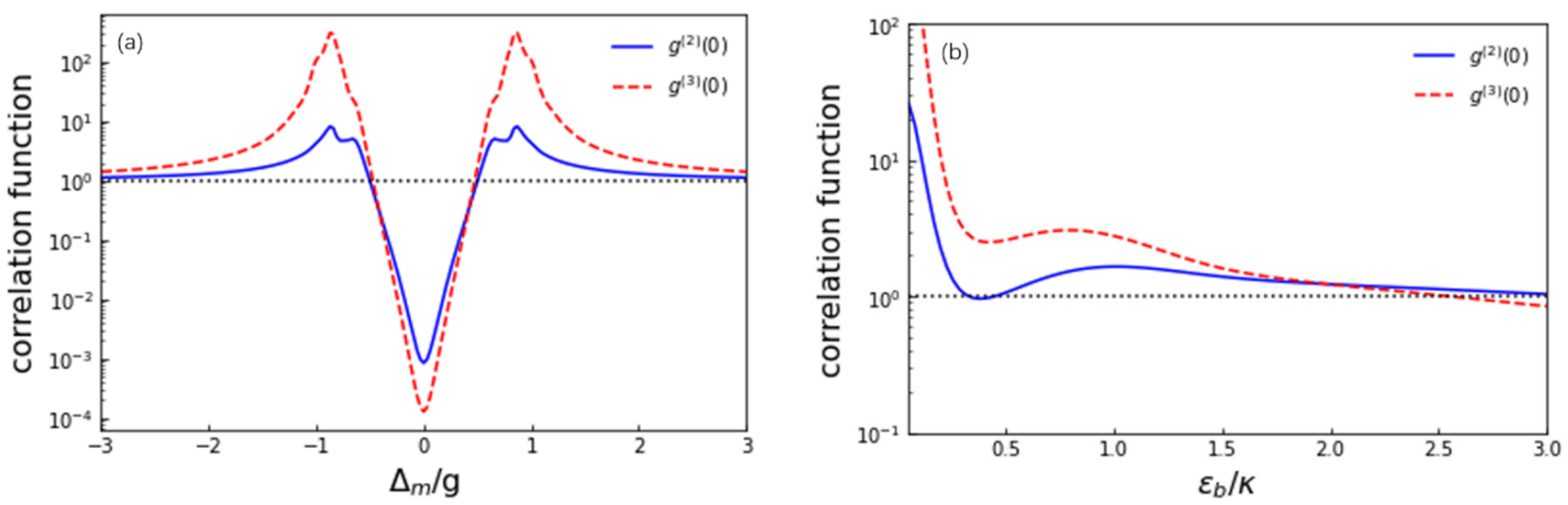

Figure 3. Steady-state equal-time second-order correlation function $g^{(2)}(0)$ and third-order correlation function $g^{(3)}(0)$ versus (a) the detuning $\Delta_{m} / g$ and (b) the driving strength $\varepsilon_{b} / \kappa$ when the mechanical resonator is weakly driven. The system parameters are $\gamma=0.01 \kappa, n_{\text {th }}=0, g=2 \kappa$, (a) $\varepsilon_{b}=0.15 \kappa$ and (b) $\Delta_{m}= \pm \frac{\sqrt{2}}{2} g$.

As depicted in Figure $4 a, b$, we plot $g^{(2)}(0)$ and $g^{(3)}(0)$ versus the detuning $\Delta_{m}^{\prime} / \kappa$ and the coupling strength $g / \kappa$. It can be seen that the values of $g^{(2)}(0)$ are always bigger than 1 when the mechanical detuning $\Delta_{m}^{\prime} / \kappa$ and the coupling strength $g / \kappa$ vary. However, the values of $g^{(3)}(0)$ can be smaller than 1 around the detuning $\Delta_{m}^{\prime}= \pm \frac{\sqrt{2}}{2} g$, as shown by the white dashed lines in Figure $4 \mathrm{~b}$. To further study the physical mechanism of the twophonon blockade effect, in Figure 5a we present $g^{(2)}(0)$ and $g^{(3)}(0)$ versus the detuning $\Delta_{m}^{\prime} / g$. It clearly shows that under the condition of the two-phonon resonant driving, i.e., $\Delta_{m}^{\prime} / g= \pm \frac{\sqrt{2}}{2}$, the curve of $g^{(2)}(0)$ has two dips with $g^{(2)}(0) \gg 1$, which indicates the two-phonon bunching. Meanwhile, one can have $g^{(3)}(0) \ll 1$ with three-phonon antibunching when $\Delta_{m}^{\prime} / g= \pm \frac{\sqrt{2}}{2}$. That is, the two-phonon blockade effect can be achieved via weakly driving the cavity field instead of the mechanical resonator, as shown by the gray region in Figure 5a. This quantum effect can also be explained from the energylevel diagram in Figure 1b. When the cavity is driven at the two-phonon resonance, i.e., $\Delta_{m}^{\prime} / g= \pm \frac{\sqrt{2}}{2}$, the ground state $|0,0\rangle$ can be transitioned to the two-phonon state $|2, \pm\rangle$, while the $|2, \pm\rangle \rightarrow|3, \pm\rangle$ transition will be suppressed due to the frequency detuning. Note that the transition $|0,0\rangle \rightarrow|0,1\rangle$ will be forbidden due to the creation of a photon accompanied by the annihilation of two phonons. In addition, in Figure $5 b$ we present the steady-state $g^{(2)}(0)$ and $g^{(3)}(0)$ as a function of the driving strength $\varepsilon_{d} / \kappa$. It is shown that with $\varepsilon_{d} / \kappa$ increases, the values of $g^{(2)}(0)$ gradually decrease and finally tends to 1 , while the values of $g^{(3)}(0)$ first decrease, then increase and finally approach 1 when $\varepsilon_{d} / \kappa$ is very large. Therefore, to obtain the two-phonon blockade effect with $g^{(2)}(0)>1$ and $g^{(3)}(0)<1$, a proper driving strength needs to be chosen. When the values of $\varepsilon_{d} / \kappa$ range from 0.055 to 0.4 , the two-phonon blockade effect can be generated, as shown by the gray region in Figure $5 b$. 

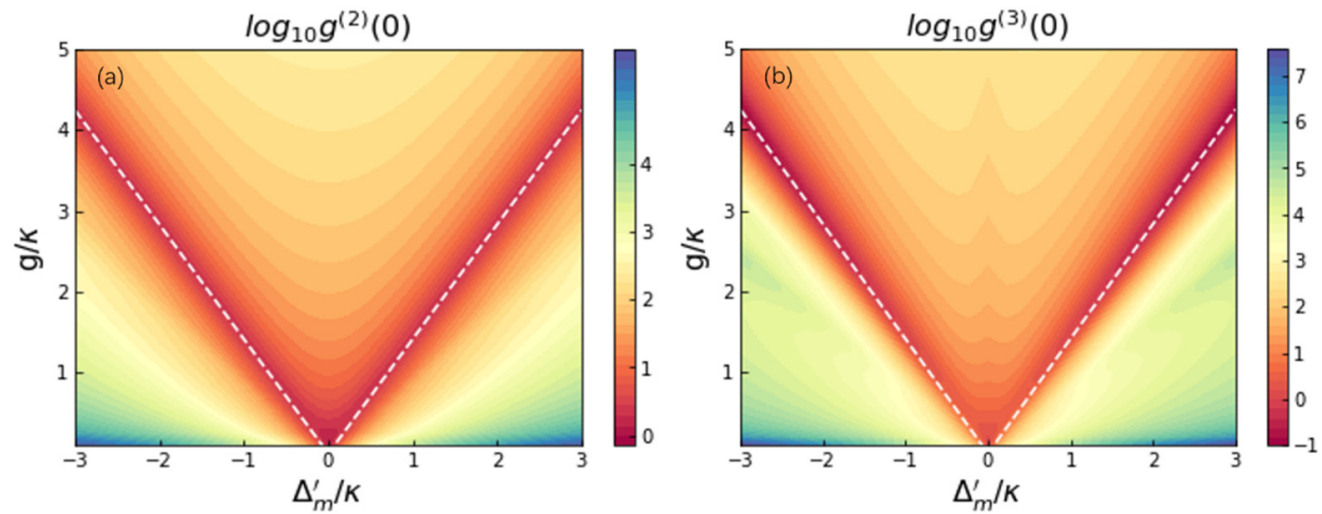

Figure 4. Steady-state equal-time (a) second-order correlation function $g^{(2)}(0)$ and (b) third-order correlation function $g^{(3)}(0)$ versus the detuning $\Delta_{m}^{\prime} / \kappa$ and the coupling strength $g / \kappa$ when the optical cavity is weakly driven. The system parameters we take are $\gamma=0.01 \kappa, \varepsilon_{d}=0.15 \kappa$ and $n_{\text {th }}=0$. The white dashed curves in (a) and (b) correspond to the cases of two-phonon resonant driving, i.e., $\Delta_{m}^{\prime}= \pm \frac{\sqrt{2}}{2} g$.
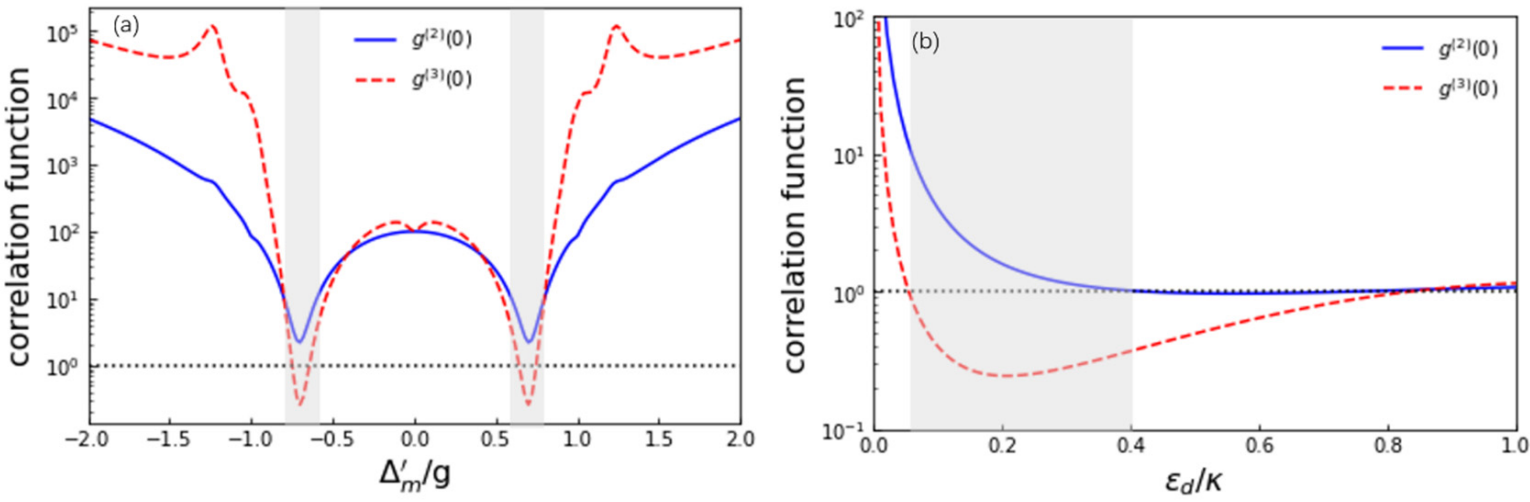

Figure 5. Steady-state equal-time second-order correlation function $g^{(2)}(0)$ and third-order correlation function $g^{(3)}(0)$ versus (a) the detuning $\Delta_{m}^{\prime} / g$ and (b) the driving strength $\varepsilon_{d} / \kappa$ when the optical cavity is weakly driven. The system parameters are $\gamma=0.01 \kappa, n_{\mathrm{th}}=0, g=3 \kappa,(\mathbf{a}) \varepsilon_{d}=0.15 \kappa$ and (b) $\Delta_{m}^{\prime}= \pm \frac{\sqrt{2}}{2} g$.

In the discussion above, we do not consider the influence of the thermal phonon occupation number on the phonon statistical characteristics, i.e., $n_{\mathrm{th}}=0$. In order to present how the two-phonon blockade effect depends on the thermal phonon number, we plot the steady-state second-order correlation function $g^{(2)}(0)$ and third-order correlation function $g^{(3)}(0)$ versus the thermal phonon number $n_{\text {th }}$, as shown in Figure $6 \mathrm{a}, \mathrm{b}$. It can be seen that when $n_{\text {th }}$ increases from $10^{-5}$ to 0.05 , the values of $g^{(2)}(0)$ are always bigger than 1 at the two-phonon resonant driving $\left(\Delta_{m}^{\prime} / g= \pm \frac{\sqrt{2}}{2}\right)$. However, with $n_{\text {th }}$ further increasing, e.g., $n_{\mathrm{th}}=0.4$ and $n_{\mathrm{th}}=10$, the values of $g^{(2)}(0)$ are approximately equal to 1 at $\Delta_{m}^{\prime} / g= \pm \frac{\sqrt{2}}{2}$. Therefore, one can find that the increase of the thermal phonon number will weaken the two-phonon bunching effect. In terms of the third-order correlation function $g^{(3)}(0)$, when $n_{\mathrm{th}}<0.4$, the values of $g^{(3)}(0)$ is smaller than 1 around the detuning $\Delta_{m}^{\prime} / g= \pm \frac{\sqrt{2}}{2}$. When $n_{\text {th }}$. increases to 10 , the values of $g^{(3)}(0)$ are bigger than 1 around $\Delta_{m}^{\prime} / g= \pm \frac{\sqrt{2}}{2}$. To clarify more clearly the influence of the thermal phonon number, the second-order correlation function $g^{(2)}(0)$ and third-order correlation function $g^{(3)}(0)$ are plotted as a function of the thermal phonon number in the case of two-phonon resonant driving in Figure 6c. It is shown that with the increase of $n_{\text {th }}$, the values of $g^{(2)}(0)$ rapidly decrease and then slowly increase, while the values of $g^{(3)}(0)$ gradually increase when $n_{\text {th }}$ increases. For the chosen system parameters, when $n_{\mathrm{th}}<0.4$ (see the inset in Figure $6 \mathrm{c}$ ), one could possibly obtain 
the two-phonon blockade effect with $g^{(2)}(0)>1$ and $g^{(3)}(0)<1$. These results indicate that to better achieve the two-phonon blockade effect, it needs to suppress the mechanical thermal noise.
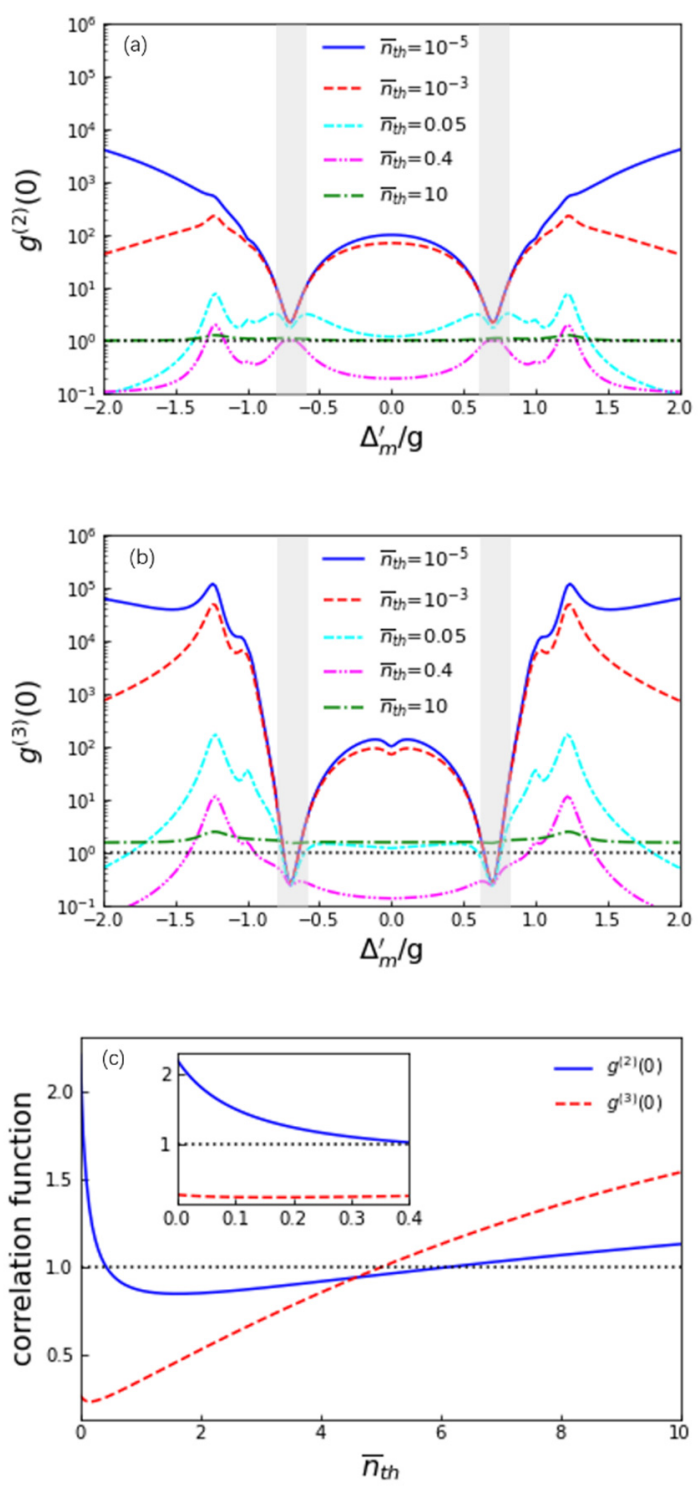

Figure 6. Steady-state equal-time (a) second-order correlation function $g^{(2)}(0)$ and (b) third-order correlation function $g^{(3)}(0)$ versus the detuning $\Delta_{m}^{\prime} / g$ for different thermal phonon numbers $n_{\text {th }}$ when the optical cavity is weakly driven. (c) The second-order correlation function $g^{(2)}(0)$ and thirdorder correlation function $g^{(3)}(0)$ versus the thermal phonon number $n_{\text {th }}$ in the case of two-phonon resonant driving. The system parameters are $\gamma=0.01 \kappa, \varepsilon_{d}=0.15 \kappa$ and $g=3 \kappa$.

Note that to obtain the two-phonon blockade effect, the condition $\omega_{m} \gg g \gg \kappa, \gamma$ needs to be satisfied, especially the strong coupling regime $g \gg \kappa$. In terms of the experimental prospect, the work in Ref. [29] demonstrates a high-finesse optical cavity with the decay rate $\kappa / 2 \pi=5 \times 10^{5} \mathrm{~Hz}$ and the quadratic optomechanical coupling $g_{0} / 2 \pi=5 \mathrm{~Hz}$. Recent advances have realized the significant enhancement of the quadratic optomechanical coupling, e.g., in a low-loss optomechanical device [30], in a fiber cavity [47], or in a photonic crystal optomechanical cavity [48]. The quadratic optomechanical coupling can be increased three orders of magnitude [30]. Based on this enhancement, the effective coupling strength $g=g_{0} \alpha$ can be much larger than the optical decay rate $\kappa$ for $\alpha \gg 1$, e.g., $\alpha \sim 10^{4}[30]$. 


\section{Conclusions}

We have explored the two-phonon blockade effect in a quadratically coupled optomechanical system, where the optical cavity is both driven by a strong pumping field and a weak driving field. A strong nonlinear interaction between the optical mode and the mechanical resonator can be induced by strongly driving the optical cavity. The effective coupling strength is tunable by adjusting the amplitude of the driving field. Based on this effectively strong nonlinearity, the two-phonon blockade effect can be achieved when a weak driving field is applied into the optical cavity. By numerically calculating the steadystate second-order and third-order correlation function, we investigated the statistical characteristics of phonons. In addition, we studied the influence of the thermal noise on the achieved two-phonon blockade effect. For comparison, we also studied the case of weakly driving the mechanical resonator, where the single-phonon blockade is generated, while the two-phonon blockade can not occur. Our work has potential applications in quantum information processing and will pave the way for the study of multiphonon quantum correlation and multiphonon quantum coherent devices.

Author Contributions: Conceptualization and model, T.-S.Y. and A.C.; numerical simulation, Z.-Y.L. and T.-S.Y.; writing—original draft preparation, Z.-Y.L.; writing—review and editing, G.-R.J., T.-S.Y. and A.C. All authors have read and agreed to the published version of the manuscript.

Funding: This research was supported by the National Natural Science Foundation of China through Grants No. 12005189, No. 12175199, and No. 12075209, Zhejiang Provincial Natural Science Foundation of China through Grant No. LZ20A040002, and the Science Foundation of Zhejiang Sci-Tech University through Grants No. 19062408-Y and No. 17062071-Y.

Conflicts of Interest: The authors declare no conflict of interest.

\section{References}

1. Aspelmeyer, M.; Meystre, P.; Schwab, K. Quantum optomechanics. Phys. Today 2012, 65, 29-35. [CrossRef]

2. Aspelmeyer, M.; Kippenberg, T.J.; Marquardt, F. Cavity optomechanics. Rev. Mod. Phys. 2014, 86, 1391-1452. [CrossRef]

3. Kippenberg, T.J.; Vahala, K.J. Cavity Optomechanics: Back-Action at the Mesoscale. Science 2008, 321, 1172-1176. [CrossRef]

4. Marquardt, F.; Girvin, S.M. Trend: Optomechanics. Physics 2009, 2, 40. [CrossRef]

5. Meystre, P. A short walk through quantum optomechanics. Ann. Phys. 2013, 525, 215-233. [CrossRef]

6. Teufel, J.D.; Donner, T.; Li, D.; Harlow, J.W.; Allman, M.; Cicak, K.; Sirois, A.J.; Whittaker, J.D.; Lehnert, K.W.; Simmonds, R.W. Sideband cooling of micromechanical motion to the quantum ground state. Nature 2011, 475, 359-363. [CrossRef]

7. Chan, J.; Alegre, T.M.; Safavi-Naeini, A.H.; Hill, J.T.; Krause, A.; Gröblacher, S.; Aspelmeyer, M.; Painter, O. Laser cooling of a nanomechanical oscillator into its quantum ground state. Nature 2011, 478, 89-92. [CrossRef]

8. Wilson-Rae, I.; Nooshi, N.; Zwerger, W.; Kippenberg, T.J. Theory of Ground State Cooling of a Mechanical Oscillator Using Dynamical Backaction. Phys. Rev. Lett. 2007, 99, 093901. [CrossRef]

9. Marquardt, F.; Chen, J.P.; Clerk, A.A.; Girvin, S.M. Quantum Theory of Cavity-Assisted Sideband Cooling of Mechanical Motion. Phys. Rev. Lett. 2007, 99, 093902. [CrossRef]

10. Corbitt, T.; Wipf, C.; Bodiya, T.; Ottaway, D.; Sigg, D.; Smith, N.; Whitcomb, S.; Mavalvala, N. Optical Dilution and Feedback Cooling of a Gram-Scale Oscillator to $6.9 \mathrm{mK}$. Phys. Rev. Lett. 2007, 99, 160801. [CrossRef]

11. Heikkilä, T.T.; Massel, F.; Tuorila, J.; Khan, R.; Sillanpää, M.A. Enhancing Optomechanical Coupling via the Josephson Effect. Phys. Rev. Lett. 2014, 112, 203603. [CrossRef]

12. Johansson, J.R.; Johansson, G.; Nori, F. Optomechanical-like coupling between superconducting resonators. Phys. Rev. A 2014, 90, 053833. [CrossRef]

13. Xuereb, A.; Genes, C.; Dantan, A. Strong Coupling and Long-Range Collective Interactions in Optomechanical Arrays. Phys. Rev. Lett. 2012, 109, 223601. [CrossRef]

14. Xuereb, A.; Genes, C.; Dantan, A. Collectively enhanced optomechanical coupling in periodic arrays of scatterers. Phys. Rev. A 2013, 88, 053803. [CrossRef]

15. Genes, C.; Xuereb, A.; Pupillo, G.; Dantan, A. Enhanced optomechanical readout using optical coalescence. Phys. Rev. A 2013, 88, 033855. [CrossRef]

16. Via, G.; Kirchmair, G.; Romero-Isart, O. Strong Single-Photon Coupling in Superconducting Quantum Magnetomechanics. Phys. Rev. Lett. 2015, 114, 143602. [CrossRef]

17. Lü, X.-Y.; Wu, Y.; Johansson, J.R.; Jing, H.; Zhang, J.; Nori, F. Squeezed Optomechanics with Phase-Matched Amplification and Dissipation. Phys. Rev. Lett. 2015, 114, 093602. [CrossRef] 
18. Bose, S.; Jacobs, K.; Knight, P.L. Preparation of nonclassical states in cavities with a moving mirror. Phys. Rev. A 1997, 56, 4175-4186. [CrossRef]

19. Mancini, S.; Man'ko, V.I.; Tombesi, P. Ponderomotive control of quantum macroscopic coherence. Phys. Rev. A 1997, 55, 3042-3050 [CrossRef]

20. Marshall, W.; Simon, C.; Penrose, R.; Bouwmeester, D. Towards Quantum Superpositions of a Mirror. Phys. Rev. Lett. 2003, 91, 130401. [CrossRef]

21. Stannigel, K.; Komar, P.; Habraken, S.J.M.; Bennett, S.D.; Lukin, M.D.; Zoller, P.; Rabl, P. Optomechanical Quantum Information Processing with Photons and Phonons. Phys. Rev. Lett. 2012, 109, 013603. [CrossRef] [PubMed]

22. Kómár, P.; Bennett, S.D.; Stannigel, K.; Habraken, S.J.M.; Rabl, P.; Zoller, P.; Lukin, M.D. Single-photon nonlinearities in two-mode optomechanics. Phys. Rev. A 2013, 87, 013839. [CrossRef]

23. Rabl, P. Photon Blockade Effect in Optomechanical Systems. Phys. Rev. Lett. 2011, 107, 063601. [CrossRef] [PubMed]

24. Kronwald, A.; Ludwig, M.; Marquardt, F. Full photon statistics of a light beam transmitted through an optomechanical system. Phys. Rev. A 2013, 87, 013847. [CrossRef]

25. Liao, J.Q.; Law, C.K. Correlated two-photon scattering in cavity optomechanics. Phys. Rev. A 2013, 87, 043809. [CrossRef]

26. Wang, H.; Gu, X.; Liu, Y.X.; Miranowicz, A.; Nori, F. Tunable photon blockade in a hybrid system consisting of an optomechanical device coupled to a two-level system. Phys. Rev. A 2015, 92, 033806. [CrossRef]

27. Xie, H.; Lin, G.W.; Chen, X.; Chen, Z.H.; Lin, X.M. Single-photon nonlinearities in a strongly driven optomechanical system with quadratic coupling. Phys. Rev. A 2016, 93, 063860. [CrossRef]

28. Nunnenkamp, A.; Børkje, K.; Girvin, S.M. Single-Photon Optomechanics. Phys. Rev. Lett. 2011, 107, 063602. [CrossRef]

29. Thompson, J.; Zwickl, B.; Jayich, A.; Marquardt, F.; Girvin, S.; Harris, J. Strong dispersive coupling of a high-finesse cavity to a micromechanical membrane. Nature 2008, 452, 72-75. [CrossRef]

30. Sankey, J.C.; Yang, C.; Zwickl, B.M.; Jayich, A.M.; Harris, J.G. Strong and tunable nonlinear optomechanical coupling in a low-loss system. Nat. Phys. 2010, 6, 707-712. [CrossRef]

31. Purdy, T.P.; Brooks, D.W.C.; Botter, T.; Brahms, N.; Ma, Z.-Y.; Stamper-Kurn, D.M. Tunable Cavity Optomechanics with Ultracold Atoms. Phys. Rev. Lett. 2010, 105, 133602. [CrossRef] [PubMed]

32. Wilson, D.J.; Regal, C.A.; Papp, S.B.; Kimble, H.J. Cavity Optomechanics with Stoichiometric SiN Films. Phys. Rev. Lett. 2009, 103, 207204. [CrossRef] [PubMed]

33. Nunnenkamp, A.; Børkje, K.; Harris, J.G.E.; Girvin, S.M. Cooling and squeezing via quadratic optomechanical coupling. Phys. Rev. A 2010, 82, 021806. [CrossRef]

34. Bhattacharya, M.; Uys, H.; Meystre, P. Optomechanical trapping and cooling of partially reflective mirrors. Phys. Rev. A 2008, 77,033819 .

35. Liao, J.-Q.; Nori, F. Photon blockade in quadratically coupled optomechanical systems. Phys. Rev. A 2013, 88, 023853. [CrossRef]

36. Seok, H.; Wright, E.M. Antibunching in an optomechanical oscillator. Phys. Rev. A 2017, 95, 053844. [CrossRef]

37. Xie, H.; Liao, C.-G.; Shang, X.; Ye, M.-Y.; Lin, X.-M. Phonon blockade in a quadratically coupled optomechanical system. Phys. Rev. A 2017, 96, 013861.

38. Xu, X.-W.; Shi, H.-Q.; Chen, A.-X.; Liu, Y.-X. Cross-correlation between photons and phonons in quadratically coupled optomechanical systems. Phys. Rev. A 2018, 98, 013821.

39. Xie, H.; Liao, C.-G.; Shang, X.; Chen, Z.-H.; Lin, X.-M. Optically induced phonon blockade in an optomechanical system with second-order nonlinearity. Phys. Rev. A 2018, 98, 023819

40. Liu, Y.-X.; Miranowicz, A.; Gao, Y.B.; Bajer, J.C.V.; Sun, C.P.; Nori, F. Qubit-induced phonon blockade as a signature of quantum behavior in nanomechanical resonators. Phys. Rev. A 2010, 82, 032101.

41. Wang, X.; Miranowicz, A.; Li, H.-R.; Nori, F. Method for observing robust and tunable phonon blockade in a nanomechanical resonator coupled to a charge qubit. Phys. Rev. A 2016, 93, 063861. [CrossRef]

42. Xu, X.-W.; Chen, A.-X.; Liu, Y.-X. Phonon blockade in a nanomechanical resonator resonantly coupled to a qubit. Phys. Rev. A 2016, 94, 063853. [CrossRef]

43. Zheng, L.-L.; Yin, T.-S.; Bin, Q.; Lü, X.-Y.; Wu, Y. Single-photon-induced phonon blockade in a hybrid spin-optomechanical system. Phys. Rev. A 2019, 99, 013804. [CrossRef]

44. Yin, T.-S.; Bin, Q.; Zhu, G.-L.; Jin, G.-R.; Chen, A. Phonon blockade in a hybrid system via the second-order magnetic gradient Phys. Rev. A 2019, 100, 063840. [CrossRef]

45. Ramos, T.; Sudhir, V.; Stannigel, K.; Zoller, P.; Kippenberg, T.J. Nonlinear Quantum Optomechanics via Individual Intrinsic Two-Level Defects. Phys. Rev. Lett. 2013, 110, 193602. [CrossRef]

46. Bin, Q.; Lü, X.-Y.; Laussy, F.P.; Nori, F.; Wu, Y. N-Phonon Bundle Emission via the Stokes Process. Phys. Rev. Lett. 2020, $124,053601$. [CrossRef]

47. Flowers-Jacobs, N.E.; Hoch, S.W.; Sankey, J.C.; Kashkanova, A.; Jayich, A.M.; Deutsch, C.; Reichel, J.; Harris, J.G.E. Fiber-cavitybased optomechanical device. Appl. Phys. Lett. 2012, 101, 221109. [CrossRef]

48. Paraïso, T.K.; Kalaee, M.; Zang, L.; Pfeifer, H.; Marquardt, F.; Painter, O. Position-Squared Coupling in a Tunable Photonic Crystal Optomechanical Cavity. Phys. Rev. X 2015, 5, 041024. [CrossRef] 